\title{
CRITERIA FOR THE EXTREMALITY OF THE KOEBE MAPPING
}

\author{
D. BSHOUTY AND W. HENGARTNER
}

(Communicated by Clifford J. Earle, Jr.)

\begin{abstract}
A criterion is developed which gives a necessary condition on a real functional in order that the Koebe Mapping be extremal for this functional in the well known class $S$ of normalized univalent functions. This is applied to the coefficient problem of $[f(z)]^{\lambda}, 0<\lambda<1$, as well as to the problem of univalence of sections of the power series expansion of $f(z)$.
\end{abstract}

\section{INTRODUCTION}

Let $H(U)$ be the linear space of analytic functions defined on the unit disk $U=\{z:|z|<1\}$ endowed with the topology of locally uniform convergence. If $f$ is analytic in an open neighborhood of the closed unit disk $\bar{U}$, then we write $f \in H(\bar{U})$. Denote by $S$ the usual class of normalized univalent functions

$$
f(z)=z+\sum_{k=2}^{\infty} a_{k}(f) z^{k}
$$

in $H(U)$. In the past, many extremal problems of the form

$$
\operatorname{Max}(\text { Min resp.) }\{F(f): f \in S\}
$$

have been successfully solved or investigated, where $F$ is a real continuous functional on $H(U)$. It has turned out that in several cases the Koebe mapping

$$
k(z)=z+\sum_{k=2}^{\infty} k z^{k}=z /(1-z)^{2}
$$

or one of its rotations $k(\eta z),|\eta|=1$, is an extremal solution for the optimization problem (1.1). Furthermore, there are still several open conjectures where it is believed that $k(z)$ is extremal. It is therefore natural to construct a variation of $k$ in $S$ in order to provide a tool to decide whether $k$ is locally extremal.

In this paper we shall present a new and much simpler proof of a powerful variation of $k$ which was developed in [1] and [2], and we give some new

Received by the editors August 15, 1989.

1980 Mathematics Subject Classification (1985 Revision). Primary 30C50.

The authors were supported in part by the Funds for the Promotion of Research at the Technion, Israel and NSERC Canada, respectively. 
applications to show how one can quickly decide whether a conjecture may hold or not. Other applications can be found in [1] and [2].

\section{A VARIATION OF THE KoEBE FUNCTION}

In this section we give a new and much simpler proof of a variation of the Koebe mapping. It involves functions $t$ which belong to the class of typically real functions

$$
T_{\mathbf{R}}=\left\{t \in H(U) ; t(0)=t^{\prime}(0)-1=0 \text { and } \operatorname{Im}\{z\} \operatorname{Im}\{t(z)\} \geq 0 \text { in } U\right\} .
$$

The following slightly weaker version of Proposition 3.1 in [2] is, in fact, rather general for the purpose of the type of applications that are considered in this paper.

Theorem 2.1. Let $h(z)$ be in $H(\bar{U})$ such that $h(0)=0$, and assume that $t(z)=[h(z)+\overline{h(\bar{z})}] / 2 \in T_{\mathbf{R}}$. Then there is a positive $\varepsilon_{0}$ and an analytic function $g(z, \varepsilon)$ in $U \times\left(0, \varepsilon_{0}\right)$ such that

$$
f_{\varepsilon}(z)=k(z)\left(1-\varepsilon h(z)+\varepsilon^{2} g(z, \varepsilon)\right)
$$

belongs to $S$ for all $\varepsilon \in\left[0, \varepsilon_{0}\right)$.

Theorem 2.1 is an immediate consequence of the following theorem.

Theorem 2.2. Let $h(z)$ be in $H(\bar{U})$ such that $h(0)=0$, and assume that $t(z)=[h(z)+\overline{h(\bar{z})}] / 2 \in T_{\mathbf{R}}$. Then there is a positive $\varepsilon_{0}$ such that

$$
k_{\varepsilon}(z)=k(z)(1-\varepsilon h(z))
$$

is univalent in $\left\{z:|z| \leq 1-c \varepsilon^{2}\right\}$ for some $c>0$ and all $\varepsilon \in\left[0, \varepsilon_{0}\right)$.

Indeed, if Theorem 2.2 holds, then, for $\rho=1-c \varepsilon^{2}$, we have

$$
\begin{aligned}
k_{\varepsilon}(\rho z) / \rho & =k\left(z-c \varepsilon^{2} z\right)\left[1-\varepsilon h\left(z-c \varepsilon^{2} z\right)\right] /\left(1-c \varepsilon^{2}\right) \\
& =\left[k(z)-c \varepsilon^{2} z k^{\prime}(z)+O\left(\varepsilon^{4}\right)\right]\left[1-\varepsilon h(z)+O\left(\varepsilon^{3}\right)\right]\left[1+c \varepsilon^{2}+O\left(\varepsilon^{4}\right)\right] \\
& =k(z)[1-\varepsilon h(z)]+c \varepsilon^{2}\left(k(z)-z k^{\prime}(z)\right)+O\left(\varepsilon^{3}\right),
\end{aligned}
$$

where $O\left(\varepsilon^{3}\right)$ is taken here with respect to the topology of locally uniform convergence.

In what follows, we are interested in the zeros of $\left[k_{\varepsilon}(z)-k_{\varepsilon}(\zeta)\right] /[z-\zeta]$.

Accordingly we define

$$
E(z, \zeta)=\left[(1-\zeta)^{2} z h(z)-(1-z)^{2} \zeta h(\zeta)\right] /[z-\zeta]
$$

Then

$$
\left[k_{\varepsilon}(z)-k_{\varepsilon}(\zeta)\right] /[z-\zeta]=[1-z \zeta-\varepsilon E(z, \zeta)] /\left[(1-z)^{2}(1-\zeta)^{2}\right] .
$$

Thus we have.to solve the equation

$$
1-z \zeta-\varepsilon E(z, \zeta)=0
$$


Let $R_{0}>1$ be such that $h \in H\left(|z| \leq R_{0}\right)$. So, $E(z, \zeta) \in H\left(|z| \leq R_{0} \times|\zeta| \leq R_{0}\right)$. Put

$$
\begin{aligned}
& r_{0}=\left(2 R_{0}-1\right)^{-1 / 2}, \\
& M_{0}=\operatorname{Max}\left\{|E(z, \zeta)|:|z| \leq R_{0} \times|\zeta| \leq R_{0}\right\}, \\
& M_{1}=\operatorname{Max}\left\{|\partial E(z, \zeta) / \partial z|:|z| \leq R_{0} \times|\zeta| \leq R_{0}\right\} \text { and } \\
& \varepsilon_{1}=\left(1-r_{0}\right)^{2} /\left(2 r_{0} M_{0}\right)
\end{aligned}
$$

and fix $\zeta$ in $\left\{\zeta: r_{0} \leq|\zeta| \leq 1\right\}$. Then, for $|z|=R_{0}$ and for all $\varepsilon \in\left[0, \varepsilon_{1}\right)$,

$$
|z \zeta-1| \geq|z \zeta|-1 \geq r_{0} R_{0}-1=\left(1-r_{0}\right)^{2} /\left(2 r_{0}\right)=\varepsilon_{1} M_{0}>|\varepsilon E(z, \zeta)| .
$$

Since $z \zeta-1=0$ has a unique solution $z=1 / \zeta$ in $\left\{z:|z|<R_{0}\right\}$, by Rouché's Theorem, we conclude that there is a unique solution $z_{\varepsilon}(\zeta)$ of $(2.3)$ in $\{z:|z|<$ $\left.R_{0}\right\}$. Moreover, we have

$$
\left|z_{\varepsilon}(\zeta)-1 / \zeta\right|=\left|\varepsilon E\left(z_{\varepsilon}(\zeta), \zeta\right) / \zeta\right| \leq \varepsilon M_{0} / r_{0},
$$

and, therefore,

$$
\begin{aligned}
\left|z_{\varepsilon}(\zeta) \zeta-1+\varepsilon E(1 / \zeta, \zeta)\right| & =\varepsilon\left|E(1 / \zeta, \zeta)-E\left(z_{\varepsilon}(\zeta), \zeta\right)\right| \\
& =\varepsilon\left|\int_{1 / \zeta}^{z_{\varepsilon}(\zeta)} \partial E(z, \zeta) / \partial z d z\right| \\
& \leq \varepsilon^{2} M_{0} M_{1} / r_{0} \equiv \varepsilon^{2} c_{1} .
\end{aligned}
$$

Finally, put $\zeta=|\zeta| \eta$. Then

$$
\begin{aligned}
|E(1 / \eta, \eta)-E(1 / \zeta, \zeta)| & =\left|\int_{|\zeta|}^{1}\{d E(1 / r \eta, r \eta) / d r\} d r\right| \\
& =\left|\int_{|\zeta|}^{1}\left[(\partial E / \partial z)\left(-1 / r^{2} \eta\right)+(\partial E / \partial \zeta) \eta\right] d r\right| \\
& \leq(1-|\zeta|) M_{1}\left(1 / r_{0}^{2}+1\right) \equiv c_{2}(1-|\zeta|) .
\end{aligned}
$$

We have proved

Lemma 2.3. Suppose that $h \in H\left(|z| \leq R_{0}\right)$ and that $h(0)=0, R_{0}>1$, $r_{0}=\left(2 R_{0}-1\right)^{-1 / 2}$ and $\varepsilon_{1}=\left(1-r_{0}\right)^{2} /\left(2 r_{0} M_{0}\right)$. Then, if $0<\varepsilon<\varepsilon_{1}$ and $r_{0} \leq|\zeta| \leq 1$, the equation (2.3) has a unique solution $z_{\varepsilon}(\zeta)$ in $\left\{z:|z|<R_{0}\right\}$. Moreover, we have

$$
\left|z_{\varepsilon}(\zeta) \zeta-1+\varepsilon E(1 / \eta, \eta)\right| \leq c_{1} \varepsilon^{2}+c_{2} \varepsilon(1-|\zeta|),
$$

where $\eta=\zeta /|\zeta|$ and the constants $c_{1}$ and $c_{2}$ are as defined above.

Before we prove Theorem 2.2, let us note that straightforward calculations give, for $|\eta|=1$,

$$
-\operatorname{Re}\{E(1 / \eta, \eta)\}= \begin{cases}{\left[2 \eta /(1+\eta)^{2}\right] \operatorname{Re}\left\{\left(1-\eta^{2}\right) t(\eta) / \eta\right\},} & \text { if } \eta \neq \pm 1 \\ 4 t^{\prime}(-1), & \text { if } \eta=-1 \\ 0, & \text { if } \eta=1,\end{cases}
$$


where $t(z)$ is defined in Theorem 2.1. In other words, $\operatorname{Re}\{E(1 / \eta, \eta)\} \leq 0$ for all $\eta$ on the unit circle $\partial U$ and equality holds only at finitely many points of $\partial U$.

Proof of Theorem 2.2. We define $c=c_{1}=M_{0} M_{1} / r_{0}$ and assume to the contrary that there exists a sequence $\varepsilon_{n}, n=1,2, \ldots$, such that $\varepsilon_{n}>0, \varepsilon_{n}$ tends to zero as $n$ tends to infinity and $k_{\varepsilon_{n}}(z)$ is not univalent in $\left\{z ;|z| \leq 1-c_{1} \varepsilon_{n}^{2}\right\}$. Since an analytic function is univalent in the closure of a simply connected Jordan domain $D$ if and only if it is univalent on the boundary of $D$, there exist two sequences $z_{n}$ and $\zeta_{n}, z_{n} \neq \zeta_{n}$, such that $\left|z_{n}\right|=\left|\zeta_{n}\right|=1-c_{1} \varepsilon_{n}^{2}$ and $k_{\varepsilon_{n}}\left(z_{n}\right)=k_{\varepsilon_{n}}\left(\zeta_{n}\right)$. Since, for sufficiently large $n$, we have $\zeta_{n} \in\left\{z: r_{0} \leq|z| \leq\right.$ $1\}$, we get, by Lemma 2.3 and the fact that $\operatorname{Re}\{E(1 / \eta, \eta)\} \leq 0$,

$$
1-2 c_{1} \varepsilon_{n}^{2}+c_{1}^{2} \varepsilon_{n}^{4}=\left|z_{n} \zeta_{n}\right|=\left|z_{\varepsilon_{n}}\left(\zeta_{n}\right) \zeta_{n}\right|
$$

$$
\begin{aligned}
& \geq\left|1-\varepsilon_{n} E\left(1 / \eta_{n}, \eta_{n}\right)\right|-c_{1} \varepsilon_{n}^{2}-c_{1} c_{2} \varepsilon_{n}^{3} \\
& \geq 1-c_{1} \varepsilon_{n}^{2}-c_{1} c_{2} \varepsilon_{n}^{3} .
\end{aligned}
$$

Therefore, we have

$$
-2 c_{1}+c_{1}^{2} \varepsilon_{n}^{2} \geq-c_{1}-c_{1} c_{2} \varepsilon_{n} .
$$

Letting $n$ tend to infinity, we get $c_{1} \leq 0$ which is a contradiction and Theorem 2.2 is proved.

\section{NeCESSARY CONDITIONS FOR THE EXTREMALITY OF THE KOEBE MAPPING}

We start this section with a general result concerning the nonextremality of the Koebe function $k(z)$ with respect to some real functional defined on $S$.

Denote by $H_{\mathbf{R}}^{0}(\bar{U})$ the set of functions $q$ in $H(\bar{U})$ such that

$$
q(z)=\sum_{k=1}^{\infty} a_{k} z^{k}, \quad a_{k} \in \mathbf{R} \text { for all } k \geq 1 .
$$

Let $F$ be a real valued functional defined on $H(U)$ which admits a Gâteaux derivative (i.e., there is a continuous linear functional $L(f ; \cdot)$ in the topological dual space $H^{\prime}(U)$ of $H(U)$ such that

$$
F(f+\varepsilon \phi+o(\varepsilon))=F(f)+\varepsilon \operatorname{Re}\{L(f ; \phi)\}+o(\varepsilon)
$$

as $\varepsilon$ tends to zero). We shall say that $F$ is $k$-real if

$$
\operatorname{Re}\{L(k(z) ; i z q(z))\}=0, \quad \text { for all } q \in H_{\mathbf{R}}^{0}(\bar{U}) .
$$

The following result holds.

Proposition 3.1. Let $F$ be a real valued functional defined on $H(U)$ admitting a Gâteaux derivative $L(f ; \cdot)$ in $H^{\prime}(U)$. If $F$ is not $k$-real then there are functions $f_{1}, f_{2} \in S$ such that $F\left(f_{1}\right)>F(k)>F\left(f_{2}\right)$. In other words, the Koebe mapping $k(z)$ cannot be extremal.

Proof. Because $F$ is not $k$-real there is a $q_{0} \in H_{\mathbf{R}}^{0}(\bar{U})$ such that

$$
\operatorname{Re}\left\{L\left(k(z) ; i z q_{0}(z)\right)\right\} \neq 0 \text {. }
$$


Fix $d \in \mathbf{R}$ and put

$$
h(z)=z-i d z q_{0}(z) / k(z) .
$$

Then $h$ satisfies the hypothesis of Theorem 2.1 and, therefore, there is an $\varepsilon_{0}>0$ and an analytic function $g(z, \varepsilon)$ in $U \times\left[0, \varepsilon_{0}\right)$ such that $f_{\varepsilon}(z)=$ $k(z)\left(1-\varepsilon h(z)+\varepsilon^{2} g(z, \varepsilon)\right)$ belongs to $S$ for all $\varepsilon \in\left[0, \varepsilon_{0}\right)$. For all $\varepsilon \in\left[0, \varepsilon_{0}\right)$, we have

$$
\begin{aligned}
F\left(f_{\varepsilon}\right) & =F(k)-\varepsilon \operatorname{Re}\{L(k ; k h)\}+o(\varepsilon) \\
& =F(k)-\varepsilon \operatorname{Re}\{L(k ; z k)\}+\varepsilon d \operatorname{Re}\left\{L\left(k ; i z q_{0}\right)\right\}+o(\varepsilon) .
\end{aligned}
$$

Suppose that Proposition 3.1 is not true. Then we have either $F\left(f_{\varepsilon}\right) \leq F(k)$ or $F\left(f_{\varepsilon}\right) \geq F(k)$ for all sufficiently small $\varepsilon$. Assume that $F\left(f_{\varepsilon}\right) \leq F(k)$. Then (3.3) reduces to

$$
-\operatorname{Re}\{L(k ; z k)\}+d \operatorname{Re}\left\{L\left(k ; i z q_{0}\right)\right\}+o(1) \leq 0 .
$$

Letting $\varepsilon$ tend to zero, we get

$$
-\operatorname{Re}\{L(k ; z k)\}+d \operatorname{Re}\left\{L\left(k ; i z q_{0}\right)\right\} \leq 0,
$$

which leads to a contradiction for some value of $d$. The other case follows analogously.

The following criteria are direct consequences of Theorem 2.1, Proposition 3.2 gives necessary conditions for $k$ to be extremal even in the class $S_{\mathbf{R}}$ of functions in $S$ having real coefficients.

Proposition 3.2. Let $F$ be a real valued functional defined on $H(U)$ admitting a Gâteaux derivative $L(f ; \cdot)$ in $H^{\prime}(U)$.

(a) If $k$ is extremal for the problem

$$
\operatorname{Max}\{F(f): f \in S\} \quad(\operatorname{Min}\{F(f): f \in S\} \text { resp. }),
$$

then

$$
\operatorname{Re}\{L(k ; k h)\} \geq 0 \quad(\operatorname{Re}\{L(k ; k h)\} \leq 0 \text { resp. })
$$

for all $h$ such that $t(z)=[h(z)+\overline{h(\bar{z})}] / 2 \in T_{\mathbf{R}}$.

(b) If $k$ is extremal for the problem

$$
\operatorname{Max}\left\{F(f): f \in S_{\mathbf{R}}\right\} \quad\left(\operatorname{Min}\left\{F(f): f \in S_{\mathbf{R}}\right\} \text { resp. }\right),
$$

then

$$
\operatorname{Re}\{L(k ; k t)\} \geq 0 \quad(\operatorname{Re}\{L(k ; k t)\} \leq 0 \text { resp. })
$$

for all $t \in T_{\mathbf{R}}$.

Proof. We assume that $\rho \in(0,1)$ and $t \in T_{\mathbf{R}}$. Then the above inequalities hold for $h(\rho z) / \rho$ and $t(\rho z) / \rho$. Letting $\rho$ tend to 1 , we deduce the result.

\section{Applications}

In this section we show how one can easily get answers to conjectures involving the Koebe mapping by applying the above results.

In the first example we consider the following problem. 
Problem 4.1. Let $s_{n}(f)$ denote the $n$th partial sum of the McLaurin expansion of an analytic function $f$. We define $r_{n}(f)=|z(0)|$ where $z(0)$ is the smallest root in absolute value of $\left[s_{n}(f)\right]^{\prime}=0$. Is it true that, for every $f \in S, s_{n}(f)$ is univalent in $\left\{z ;|z|<r_{n}(k)\right\}$ ?

An account of the history of this problem is found in $[3, \S 8.2$, p. 246]. The answer was believed to be affirmative in view of the partial results in [7] and [3, Example 7, p. 272].

Applying Proposition 3.1 we show that the answer to this question is negative for $n=3$. Consider the real valued functional $F_{n}(f)$ defined on $H(U)$ by

$$
F_{n}(f)=\operatorname{Min}\left\{|z|:\left[s_{n}(f)\right]^{\prime}(z)=0\right\}=r_{n}(f) .
$$

Let $z(\varepsilon)$ satisfy $\left[s_{n}(k+\varepsilon \phi)\right]^{\prime}(z(\varepsilon))=0$ and $|z(\varepsilon)|=r_{n}(k+\varepsilon \phi)$. Then we have

$$
\left[s_{n}(k+\varepsilon \phi)\right]^{\prime}(z(\varepsilon))=\left[s_{n}(k)\right]^{\prime}(z(\varepsilon))+\varepsilon\left[s_{n}(\phi)\right]^{\prime}(z(\varepsilon))=0 .
$$

If $\left[s_{n}(k)\right]^{\prime \prime}(z(0)) \neq 0$, then, by the Implicit Function Theorem, we know that $z(\varepsilon)$ is analytic in $\varepsilon$ and admits the expansion

$$
z(\varepsilon)=z(0)+\varepsilon a+O\left(\varepsilon^{2}\right)
$$

in a neighborhood of the origin. The factor $a$ can be explicitly expressed by

$$
a=-\left[s_{n}(\phi)\right]^{\prime}(z(0)) /\left[s_{n}(k)\right]^{\prime \prime}(z(0)) .
$$

Hence,

$$
F_{n}(k+\varepsilon \phi)=\left|z(0)+\varepsilon a+O\left(\varepsilon^{2}\right)\right|=|z(0)|+\varepsilon|z(0)| \operatorname{Re}\{a / z(0)\}+O\left(\varepsilon^{2}\right)
$$

so that

$$
L_{n}(k ; \phi)=-|z(0)| \cdot\left[s_{n}(\phi)\right]^{\prime}(z(0)) /\left\{z(0)\left[s_{n}(k)\right]^{\prime \prime}(z(0))\right\} .
$$

We now show that, for $n=3, L_{3}(k ; \cdot)$ is not $k$-real. We have $z(0)=$ $(-2 \pm i \sqrt{5}) / 9$ and $\left[s_{3}(k)\right]^{\prime \prime}(z(0))= \pm 2 \sqrt{5} i \neq 0$. Take, in $(3.1), q(z)=z$. Then

$$
\operatorname{Re}\left\{L_{3}(k(z) ; i z q(z))\right\}=\operatorname{Re}\left\{L_{3}\left(k(z) ; i z^{2}\right\}= \pm 1 /(3 \sqrt{5}) \neq 0,\right.
$$

which shows that the answer to the question in Problem (4.1) is negative for $n=3$.

Numerical calculations indicate that $\left[s_{n}(k)\right]^{\prime \prime}(z(0))$ is not real if $n$ is odd, which implies that the conjecture fails for $S$ in these cases. For even $n, z(0)$ is real and we were unable to find any counterexamples. We therefore modify Problem 4.1:

Conjecture. Let $r_{n}(f)$ be defined as in (4.1). Then

(a) $r_{n}(f) \geq r_{n}(k)$ for every $f \in S_{\mathbf{R}}$;

(b) $r_{2 n}(f) \geq r_{2 n}(k)$ for every $f \in S$. 
In the next application we consider the following problem:

Problem 4.2. Let $f$ be in $S$. For which positive $\lambda$ does the Koebe mapping $k(z)$ maximize $\operatorname{Re}\left\{a_{n}\left([f / z]^{\lambda}\right)\right\}$ ?

It is an immediate deduction from the De Branges proof of the Milin conjecture that, for $\lambda \geq 1$,

$$
\left|a_{n}\left([f / z]^{\lambda}\right)\right| \leq a_{n}\left([k / z]^{\lambda}\right)
$$

(see also [5] and [6]). Grinshpan [4] proved that (4.2) fails to be true for all even coefficients, if $0<\lambda<1$, and for all odd coefficients, if $0<\lambda<1 / 2$. The case $\lambda=1 / 2$ was disproved by Schaeffer and Spencer [8]. We first show that these last results are simple applications of Proposition 3.2. Define

$$
F_{n}(f)=\operatorname{Re}\left\{a_{n}\left([f / z]^{\lambda}\right)\right\}, \quad f \in S .
$$

Then, for $t \in T_{\mathbf{R}}$,

$$
\begin{aligned}
F_{n}(k+\varepsilon k t) & \left.=\operatorname{Re}\left\{a_{n}([k+\varepsilon k t) / z]^{\lambda}\right)\right\}=\operatorname{Re}\left\{a_{n}\left([k / z]^{\lambda}\left(1+\varepsilon \lambda t+O\left(\varepsilon^{2}\right)\right)\right\}\right. \\
& =F_{n}(k)=\varepsilon \lambda \operatorname{Re}\left\{a_{n}\left(t[k / z]^{\lambda}\right)\right\}+O\left(\varepsilon^{2}\right) .
\end{aligned}
$$

Hence, $L(k ; k t)=\lambda a_{n}\left(t[k / z]^{\lambda}\right)$. By Proposition 3.2, the Koebe mapping is not extremal for Problem (4.2), if we can find a function $t \in T_{\mathbf{R}}$ such that

$$
\operatorname{Re}\left\{a_{n}\left(t[k / z]^{\lambda}\right)\right\}=a_{n}\left(t[k / z]^{\lambda}\right)<0 .
$$

Now take $t(z)=z /\left[(1-z)^{1-\lambda+\delta}(1+z)^{1+\lambda-\delta}\right]$ for some small real $\delta$. Then, since $t$ has real coefficients and is a univalent starlike mapping, we conclude that $t \in T_{\mathbf{R}}$. For the even coefficients $(n=2 m)$ we have

$$
\begin{aligned}
a_{2 m}\left(t[k / z]^{\lambda}\right) & =a_{2 m}\left(\left[z /\left(1-z^{2}\right)^{\lambda+1}\right][(1+z) /(1-z)]^{\delta}\right) \\
& =a_{2 m}\left(\left[z /\left(1-z^{2}\right)^{\lambda+1}\right]\left[1+\delta \log \{(1+z) /(1-z)\}+O\left(\delta^{2}\right)\right]\right) \\
& =\delta \cdot a_{2 m}\left(\left[z /\left(1-z^{2}\right)^{\lambda+1}\right] \log [(1+z) /(1-z)]\right)+O\left(\delta^{2}\right) \\
& \equiv \delta \cdot a_{2 m}(\Psi(z))+O\left(\delta^{2}\right), \quad \text { say. }
\end{aligned}
$$

Note that all the even coefficients of $\Psi(z)$ are positive, so that, for some small negative $\delta$ (4.4) holds. Therefore, we have shown that, for even $n$ and for all $\lambda, 0<\lambda<1$, the Koebe mapping is not extremal, even in $S_{\mathbf{R}}$.

Consider now the case of odd coefficients. Since $\Lambda_{n}(t) \equiv a_{n}\left(t[k / z]^{\lambda}\right)$ is a continuous linear functional on $T_{\mathbf{R}}$ and since we are interested in a negative value of $\operatorname{Min}\left\{\Lambda_{n}(t): t \in T_{\mathbf{R}}\right\}$, it is enough to consider the extreme points of $T_{\mathrm{R}}$ :

$$
t_{\vartheta}(z)=z /\left[\left(1-e^{i \vartheta} z\right)\left(1-e^{-1 \vartheta} z\right)\right]=z+\sum_{k=2}^{\infty}\left[\sin (k \vartheta) z^{k}\right] / \sin (\vartheta), \quad 0 \leq \vartheta \leq 2 \pi
$$


The coefficients of

$$
[k / z]^{\lambda}=1+\sum_{k=1}^{\infty} c_{k}(\lambda) z^{k}
$$

are given by

$$
c_{k}(\lambda)=\left[\prod_{j=0}^{k-1}(2 \lambda+j)\right] / k !
$$

and satisfy the recursion formula

$$
c_{k+1}(\lambda)=(2 \lambda+k) c_{k}(\lambda) /(k+1), \quad c_{0}(\lambda)=1 .
$$

Hence,

$$
\Lambda_{n}\left(t_{\vartheta}\right)=a_{n}\left(t_{\vartheta}[k / z]^{\lambda}\right)=\sum_{k=1}^{n}[\sin (k \vartheta) / \sin (\vartheta)] c_{n-k}(\lambda) .
$$

First, fix $\lambda$ in $(0,1 / 2)$ and put $n=2 m+1$. Then the sequence $\left\{c_{k}(\lambda)\right\}$ decreases to zero. If $\vartheta=2 \pi /(2 m+1)$, then (4.8) becomes

$$
\Lambda_{2 m+1}\left(t_{\vartheta}\right)=\sum_{k=1}^{m}[\sin (k \vartheta) / \sin (\vartheta)]\left[c_{2 m+1-k}(\lambda)-c_{k}(\lambda)\right]<0 .
$$

Therefore, the Koebe mapping is not extremal for these cases. For the case $\lambda=1 / 2$, put $\vartheta=\pi(2 n+1) /(n(n+1))$. Then we have

$$
\Lambda_{n}(\vartheta)=[\sin ((n+1) \vartheta / 2) \cdot \sin (n \vartheta / 2)] /[\sin (\vartheta) \sin (\vartheta / 2)]<0,
$$

and again the Koebe mapping is not extremal.

Next we study the cases $\lambda \in(1 / 2,1)$. Let $\lambda_{n}$ be defined by

$$
\lambda_{n}=\sup \left\{\lambda: a_{n}\left(t[k / z]^{\lambda}\right)<0, \quad \text { for some } t \in T_{\mathbf{R}} .\right.
$$

Then we have, for each $n, 1 / 2<\lambda_{n} \leq 1$ and $\lambda_{n}=1$ for all even $n$. On the other hand, Grinshpan [4] has shown that $\lambda_{3}<.84$. We are interested in giving a lower bound for $\lambda_{n}$ by applying Proposition 3.2.

Using the fact that $\Lambda_{n}\left(t_{\theta}\right)$ is an even function of $t$ and that $\sin t \geq 0$ on $[0, \pi]$, we may restrict ourselves to the solution of the mathematical program

$$
\operatorname{Sup}\left\{\lambda>0: \operatorname{Inf}\left\{\sum_{k=1}^{2 m+1} \sin (k \vartheta) c_{2 m+1-k}(\lambda): 0<\vartheta<\pi\right\}<0\right\} \text {. }
$$

First, let us give a lower bound for $\lambda_{3}$. The minimum of

$$
\begin{aligned}
& \{\lambda(2 \lambda+1) \sin (t)+2 \lambda \sin (2 t)+\sin (3 t)\} / \sin (t) \\
& \quad=\lambda(2 \lambda+1)+4 \lambda \cos (t)+4 \cos ^{2}(t)-1, \quad 0 \leq t<2 \pi,
\end{aligned}
$$

is attained for $\cos (t)=-\lambda / 2$ which leads to the estimate $(\sqrt{5}-1) / 2 \leq \lambda_{3}$. The following table contains lower bounds for $\lambda_{n}$ which were obtained from (4.9) 
by numerical methods:

$$
\begin{aligned}
& \lambda_{3} \geq .618 ; \quad \lambda^{5} \geq .672 ; \quad \lambda_{7} \geq .704 ; \quad \lambda_{9} \geq .725 ; \quad \lambda_{11} \geq .741 ; \\
& \lambda_{13} \geq .753 ; \quad \lambda_{15} \geq .762 ; \quad \lambda_{17} \geq .770 ; \quad \lambda_{19} \geq .777 ; \quad \lambda_{21} \geq .782 ; \\
& \lambda_{31} \geq .802 ; \quad \lambda_{51} \geq .824 ; \quad \lambda_{101} \geq .847 \text {. } \\
& \text { REFERENCES }
\end{aligned}
$$

1. D. Bshouty and W. Hengartner, Local behaviour of coefficients in subclasses of $S$, Contemp. Math. Amer. Math. Soc. 38 (1985), 77-84.

2. $\frac{,}{\text { A }}$ variation of the Koebe mapping in a dense subset of $S$, Canad. J. Math. 39 (1987), 54-73.

3. P. L. Duren, Univalent functions, Springer-Verlag, New York and Berlin, 1983.

4. A. Z. Grinshpan, On the power stability for the Bieberbach inequality, Analytic Number Theory and the Theory of Functions, 5, Zap. Nauchn. Sem. Leningrad. Otdel. Mat. Inst. Steklov. (LOMI) 125 (1983), 58-64.

5. A. Z. Grinshpan and I. M. Milin, Logarithmic coefficients means of univalent functions, Complex Variables Theory Appl. 7 (1986), 139-147.

6. W. K. Hayman and J. A. Hummel, Coefficients of powers of univalent functions, Complex Variables Theory Appl. 7 (1986), 51-70.

7. M. S. Robertson, The partial sums of multivalently starlike functions, Ann. of Math. $\mathbf{4 2}$ (1941), 829-838.

8. C. Schaeffer and D. C. Spencer, Coefficients of Schlicht functions, Duke Math. J. 10 (1943), 611-635.

Department of Mathematics, Technion, Haifa, IsRael

Departement de Mathematiques, Universite laval, Québec, G1K 7P4 Canada 